\title{
WOLF-RAYET STELLAR WIND THEORY
}

\author{
J. P. CASSINELLI \\ Department of Astronomy \\ University of Wisconsin-Madison \\ 475 N. Charter Street \\ Madison, WI 53706
}

\begin{abstract}
Two possible solutions to the Wolf-Rayet wind momentum problem are discussed: purely radiation driven wind theory, with multi-line effects, and Luminous Magnetic Rotator theory. Several recently developed radiative processes for enhancing $\stackrel{M}{M}$ or $v_{\infty}$ are described, and it is concluded that only the winds of rather hot luminous WolfRayet stars could possibly be driven by radiation. These stars should show evidence of acceleration at large radial distances. For the rapid rotators, it is possible to drive a dense equatorial outflow. Limits are discussed regarding the needed surface magnetic fields. With this model, the wind momentum problem is solved in a piece-wise fashion by having the large radio flux of Wolf-Rayet stars come from the equatorial zone and the broad P Cygni lines, arising in the polar wind. The Luminous Magnetic Rotator model can also be tested through observation, primarily through spectropolarimetry.
\end{abstract}

\section{Introduction}

This paper will focus on the most perplexing aspect of Wolf-Rayet winds - the wind momentum problem. Cassinelli and Castor (1973) derived an expression for the maximum mass loss rate that could be driven by the transfer of the stellar radiative momentum: $\stackrel{M}{\max }=\mathrm{L} / \mathrm{v}_{\infty} \mathrm{c}$. This is called the "single scattering maximum" because it assumes every stellar photon transfers its momentum, $\mathrm{hv} / \mathrm{c}$, just once. The ratio of the actual mass loss rate to $\dot{M}_{\text {max }}$ is given by the "efficiency factor for ejecting matter by radiation", $\eta$, where

$$
\eta=\frac{\dot{\mathrm{M}} \mathrm{v}_{\infty}}{\mathrm{L} / \mathrm{c}}
$$

In the period 1979-1981, it became clear that Wolf-Rayet stars posed a serious problem, for their $\eta$ values were found to be in the range $\eta=3$ to 30 (Barlow, Smith and Willis 1981). That is, there is about one order of magnitude more momentum in the wind than in the radiation field that is supposed to have driven it. (Current estimates of $\eta$ are given by Schmutz et al. 1989 and are in the range, 5 to 74). The initial reaction by most wind theorists was that surely the luminosity or stellar temperature had been underestimated. However, after a general consensus was reached that the momentum problem was real, two approaches were followed in attempting to explain the problem: (1) to modify and 
improve radiation driven wind theory to extract more momentum from the radiation field, or (2) to invoke "other" forces or non-spherical effects that could drive a fast wind. In this review I will first discuss the significant improvements that have been made over the past decade in line driven wind theory. Then I will discuss the "Luminous Magnetic Rotator" model that gives rise to a non-spherically symmetric picture for Wolf-Rayet stars.

\section{Purely Radiation Driven Winds}

It is convenient to focus on the two factors in the wind momentum rate product, $\dot{M} v_{\infty}$. The Wolf-Rayet stars have "massive" winds and "fast" winds. As a background theme for discussing and classifying the nature of recent developments regarding $M$ and $v_{\infty}$, it is helpful to use the Leer and Holzer (1980) "laws of wind theory":

(I) If additional Momentum or energy is added only to the supersonic (super critical point) region of the outflow, there will only be an increase in the terminal velocity but no increase in the mass loss rate.

(II) If momentum or energy are added to the sub-critical point region, there will be an

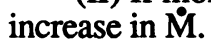

Let us illustrate these two laws using simple radiation driven wind theory in which we modify the value of $\Gamma$, the ratio of the radiative acceleration to gravity

$$
\Gamma=g_{R a d} / g=k_{F} L /(4 \pi c G M)
$$

and solve the wind momentum equation,

$$
v \frac{d v}{d r}+\frac{1}{\rho} \frac{d p}{d r}+\frac{G M}{r^{2}}(1-\Gamma)=0
$$

The results are shown in Figure 1. The top panel shows the nominal model, with no modifications in $\Gamma$. The second panel shows the effect of modifying $\Gamma$ in the supersonic region, note that only $v(r)$ changes. The last panel shows the effect of having some momentum addition occurring in the subsonic region. Note that now both $\dot{M}$ and $v(r)$ change, as does the location of the critical point. The reason the mass loss rate changes when there is additional deposition deep in the wind is that the subsonic region is nearly hydrostatic and the deposition increases the scale height of the density distribution. Therefore the product of $4 \pi \rho_{s} a r_{s}^{2}=\dot{M}$, where a, is the sound speed, can increase greatly because of the change in density $\rho_{s}$ at the critical point. With this basic result of wind theory, let us now explore four radiation related effects that tend to modify either the deposition deep in the wind (and hence increase $\dot{M}$ ), or the deposition far out in the wind (and hence increase $v_{\infty}$ ):

(1) The finite cone angle correction factor, or "Modified CAK" theory as developed by Friend and Castor (1983); Pauldrach, Puls and Kudritzki (1986); Friend and Abbott (1986).

(2) The "multi line" or multiple scattering effects of Panagia et al. (1981); Friend and Castor (1983); Abbott and Lucy (1986); and Puls (1987).

(3) Anisotropic line diffuse radiation field effect as explored by Puls and Hummer

(4) The increase in continuum optical depth as the star evolves at constant $\dot{M}, L$ to smaller radius and the accompanying effects of enhanced line opacity (Conti and Abbott 1987). 


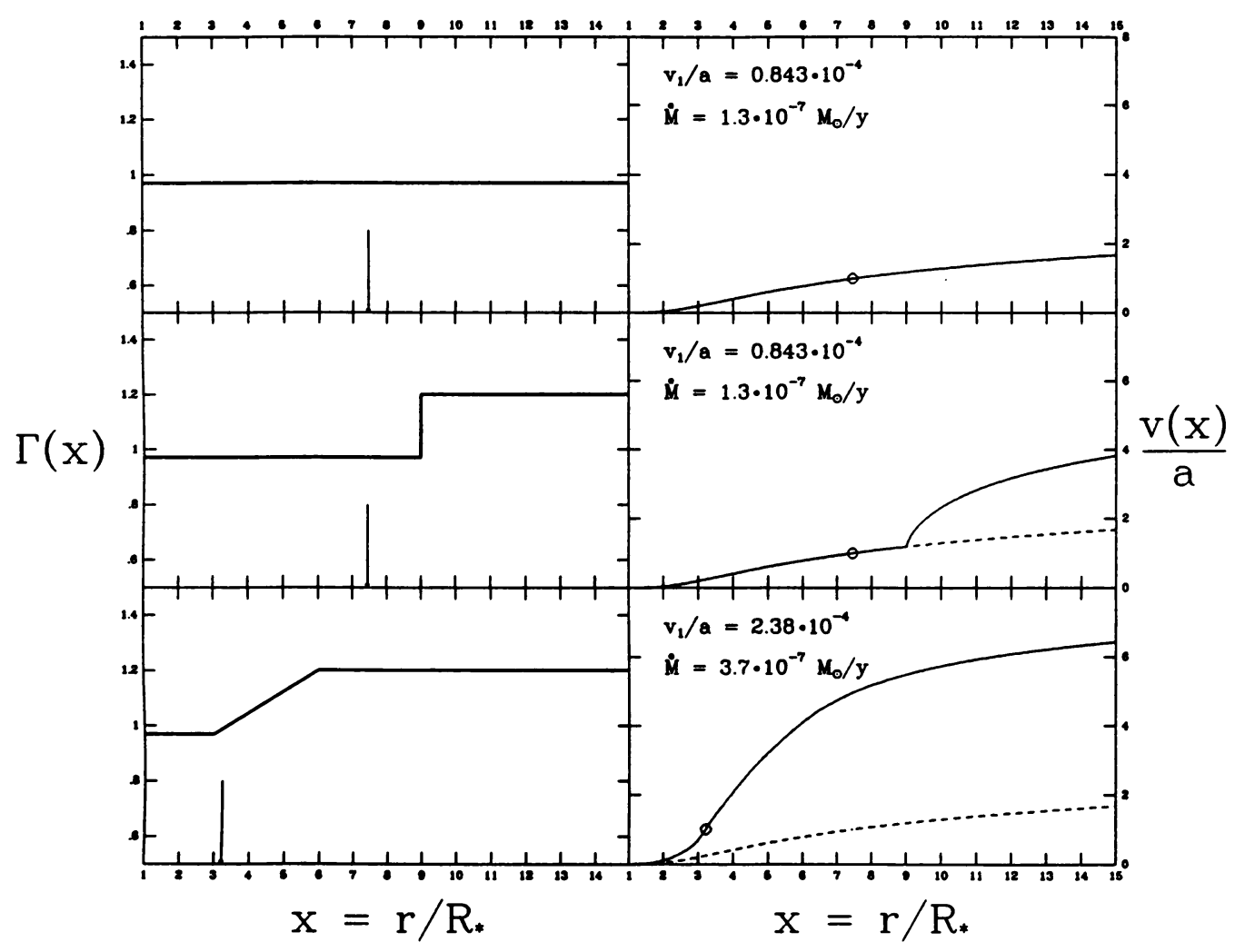

Figure 1. The left panels show three distributions of the radiation acceleration/gravity ratio, $\Gamma$, versus radial distance. The arrow indicates the location of the wind's sonic point. The right panels show the corresponding velocity $\mathrm{v}$ versus radial distance, with the sonic point marked on the curves. The velocity at the base of the wind, $v_{1}$, and the mass loss rate $M$, are also shown. The top figure shows the nominal velocity law, i.e. with no change in $\Gamma$. The second set of figures shows the effect of adding momentum to the supersonic portion of the flow alone, and it is to be noted that only the velocity law is changed relative to the nominal case, but not the mass loss rate. In the bottom figure, momentum is added to both the subsonic and supersonic part of the flow and now both $v(r)$ and $M$ change. (Figure prepared by D. van den Berk).: 
The relative importance of these four effects in regards to the momentum problem will be discussed at the end of this section. There we will also discuss the limitations on the two laws of Leer and Holzer.

\subsection{MODIFIED CAK LINE DRIVEN WIND THEORY}

The classic papers on line driven wind theory are those of Lucy and Solomon (1970) and Castor, Abbott, and Klein (1975). Lucy and Solomon showed that one line (C IV 1550 located at the peak of the spectral energy distribution of an $\mathrm{OB}$ supergiant could drive a wind with $\stackrel{M}{M}=10^{-8} \mathrm{M} / \mathrm{yr}$ to speeds of $3000 \mathrm{~km} / \mathrm{sec}$. The velocity is about right but the mass loss rate is too low. Castor, Abbott, and Klein (hereafter CAK) developed a clever approach for treating thousands of lines. They found that a wind with $\mathbf{M} \geq \dot{M}_{\text {observed }}$ and $\mathrm{v}_{\infty} \approx 1.1$ vescape could be driven. Their terminal velocity was too small, as observations have $v_{\infty} \approx 2-4 x$ vescape. A straight-forward modification of the CAK theory led to drastic improvements in the comparison between the theoretical predictions and the observations. This modification was the incorporation of the finite cone correction factor. In the original CAK paper and subsequent papers by Abbott (1982) it had been assumed that the radiation field was radial, as if coming from a point source. The radial assumption has two effects on the line acceleration. A smaller stellar luminosity produces a larger flux and hence larger acceleration. The radial approximation leads to an over-estimate of the penetration probability $\beta=\left(1-\mathrm{e}^{-\tau / \tau)}\right.$ that is used to calculate the radiative acceleration in Sobolev theory. With the non-radial incident radiation field used in the modified CAK theory, the available stellar luminosity produces less acceleration close to the star, and the penetration probability, $\beta$, is reduced in regions close to the star. As an immediate consequence, the predicted mass loss rates were reduced by $\sim$ factor of $2-3$, bringing them more in line with observations of $\mathrm{O}$ and $\mathrm{OB}$ stars. Farther out, in the supercritical regions the radiation field is indeed nearly radial as in the CAK models and since a smaller mass is being driven, a faster flow can thereby result. Friend and Abbott (1986) and Pauldrach, Puls and Kudritzki (1986) show that there is now excellent agreement between theory and observation for most $\mathrm{O}$ and $\mathrm{B}$ stars.

Now, as for Wolf-Rayet stars, Pauldrach et al. (1985) surprised many of us by showing that modified CAK theory could explain the wind of the well studied Wolf-Rayet star V444Cyg, which has a momentum efficiency factor of $\eta \approx 3$. Subsequently, Friend, Poe and Cassinelli (1987) studied this ability of modified CAK theory to produce $\eta$ factors greater than unity, and their results are shown in Figure 2. This is a plot of $\dot{M}$ vs. $\mathrm{V}_{\infty}$, it shows the locations of three Wolf-Rayet stars, as well as results of modified CAK theory for the noted values of $M, R$, and $\Gamma_{\text {es }}$.

Several things should be noted in regards to Figure 2. (a) The $\dot{M}$ and $v_{\infty}$ data for the three stars can be fitted only by using rather large values for $\Gamma_{\mathrm{es}}=\sigma_{\mathrm{es}} \mathrm{L} / 4 \pi \mathrm{c} \mathrm{GM}$; ranging from about 0.65 for V444 Cyg to values of about 0.88 for $\gamma^{2}$ Vel. These are unacceptable values for $\Gamma_{\text {es }}$ because the value derived from theoretical interior models is only about 0.4 . So a new rule has been added to the game: wind theorists should only use values of $\Gamma_{\text {es }}$ allowed by interior considerations. (b) Note that V444 Cyg has a rather mild momentum problem as compared to the other two stars shown, so that an explanation of the wind momentum of this star does not mean that a solution of "the Wolf-Rayet problem" has been found. (c) The figure indicates that all three stars could be fit using some (albeit unacceptable value of $\left.\Gamma_{\mathrm{es}}\right)$. Why is this? The reason is that a basic assumption in CAK and modified CAK theory is simply not valid. Recall that in CAK theory, (see Abbott, 1988) the force on a "resonance shell" is calculated as if the shell receives the full unattenuated stellar intensity. The models ignore the scattering or absorption by line resonance shells that lie closer to the star, i.e. "line overlap" is not accounted for. Therefore the CAK theories tend to overestimate the momentum deposition and the fit to 


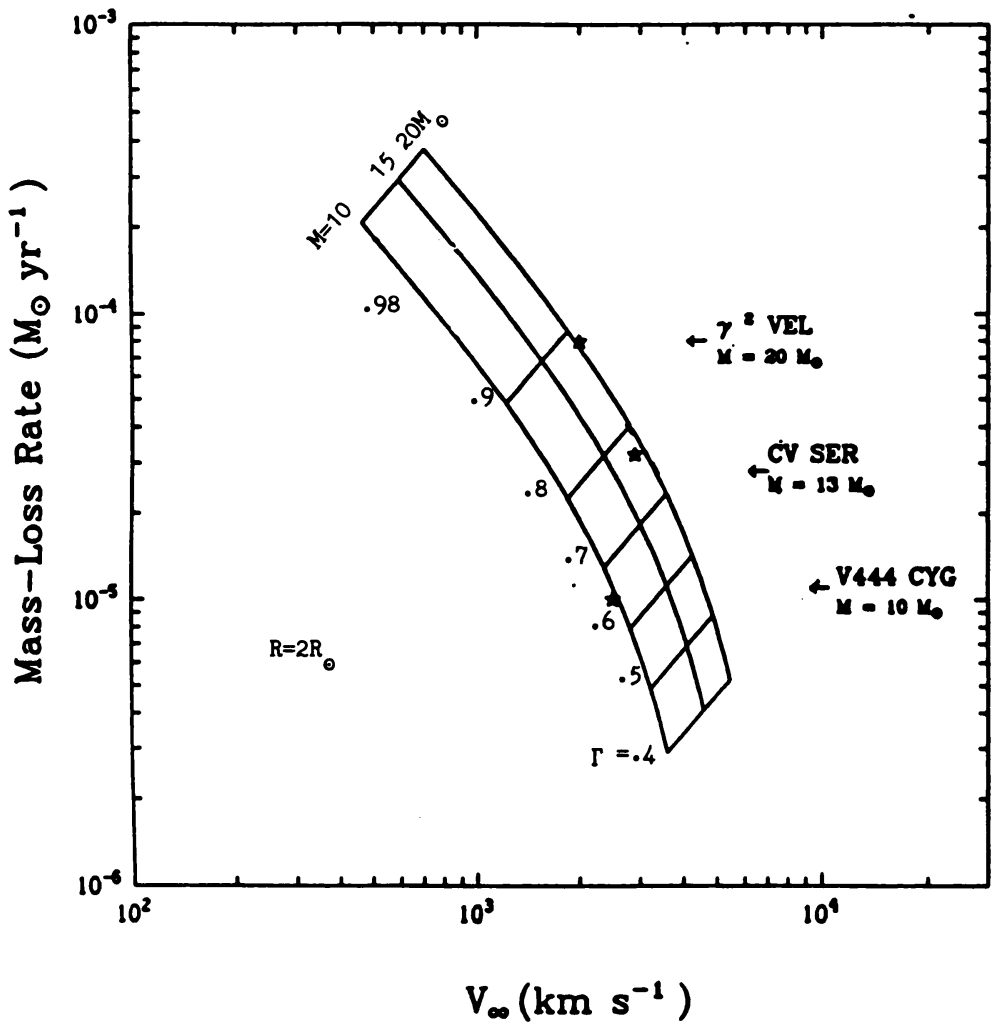

Figure 2. A plot of mass loss rate versus wind terminal velocity. The symbols show the values for $\mathcal{M}$, and $v_{\infty}$ for three Wolf-Rayet stars. The grid shows results of the "modified-CAK" models of Friend, Poe, and Cassinelli (1987). These models were carried out for a star with radius equal to 2 solar radii, and for the seven values of $\Gamma$ and three values for the stellar mass as indicated. The calculations used the CAK parameters $k=0.3$ and $\alpha=0.6$ (Abbott 1982). 
the stellar data shown in Figure 2 is not valid. There is a need to account for "line overlap" considerations or multi-line effects, discussed below.

\subsection{MULTILINE EFFECTS}

Overlapping spectral lines are those that are separated by less than the Doppler range in frequencies through the wind, i.e. $\Delta v<\Delta v_{\text {wind }}=2 v_{0} v_{o} / c$. We have discussed how the lines could reduce the momentum deposition at some radius because of scattering deeper in the wind, however, overlapping lines can also increase the net momentum deposition! This can occur in two ways: (a) Multiple scattering which can lead to a multiple deposition of photon momentum $\mathrm{hv} / \mathrm{c}$, and (b) Thermal emission of a line photon from a shell closer to the star produces photons that can be scattered by atoms farther out in the wind. Both effects have been studied by Puls (1987), here we will discuss only the effects of multiple scattering. The multiple scattering was explored for a statistical distribution of lines by Friend and Castor (1983) who found that there could be an increase in the total momentum deposition by a factor of 3-5. Abbott and Lucy (1986) used a realistic line distribution versus frequency, assumed only scattering occurs (no absorptions), and followed the momentum deposition using a Monte Carlo method. They assumed a velocity law, and used the computed energy deposition to derive a mass loss rate. Their result for the O4f star $\zeta$ Pup is shown in Figure 3. Note that there are a number of very short steps which tend to deposit photon momentum about equally in the forward and backward direction. However, there are also several very long mean free paths. Because of a long mean free path a photon scattered in a inward direction can encounter its next scattering while again having a net outward component. With this occuring, a photon can deposit more than one times $\mathrm{hv} / \mathrm{c}$. The deposition history is shown in Figure 4, where we see that for the $\zeta$ Pup model the escaping photon has deposited about $4 \times \mathrm{xv} / \mathrm{c}$. For a Wolf-Rayet star, it is likely that this multiplying factor could be even larger for reasons discussed in section 2.4. Where is this extra force coming from, and is there any limit? During the scattering processes in an expanding envelope, not only is there a shift in photon direction but also a red shift of the photon, an energy $h \Delta v$ is transferred to the flow by this redshift. Consider then the wind energy flow or wind luminosity

$$
\mathrm{L}_{\mathbf{w}} \equiv \frac{1}{2} \dot{\mathrm{M}} \mathrm{v}_{\infty}^{2}
$$

on using equation 1 , this becomes:

$$
\begin{aligned}
\mathrm{L}_{\mathrm{w}} & =\frac{1}{2} \eta \mathrm{L} \frac{\mathrm{v}_{\infty}}{\mathrm{c}} \\
& \approx \frac{.01}{2} \mathrm{~L}_{*} \eta
\end{aligned}
$$

So for WR stars with $\eta=3$ to 30 , we have $L_{w}=1.5$ to 15 percent of the stellar luminosity. Which means the average photon is required to redshift up to as much as $15 \%$. This is certainly possible in principle, but has not yet been demonstrated by a detailed model.

The multi-line effects increase the momentum deposition rather far from the star, $3 \mathrm{R} *$ $<\mathrm{r}<50 \mathrm{R} *$, and there is some observational evidence (Koenigsberger, 1990) that WR 


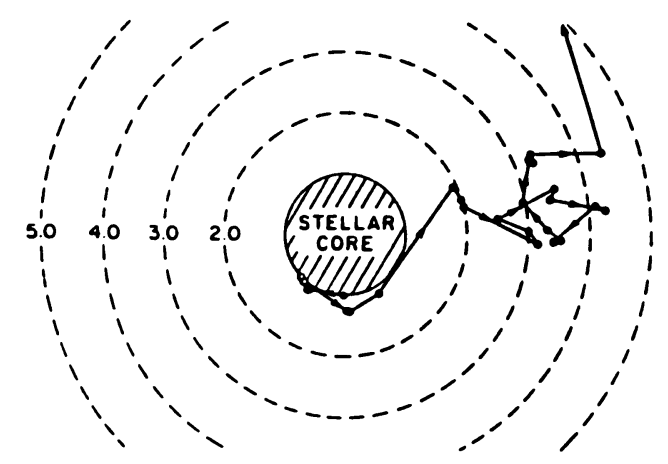

Figure 3. Shows the path of a photon through the wind of \Pup (O4f), experiencing multi-scatterings. The path is shown projected onto a plane. (Abbott and Lucy 1985).

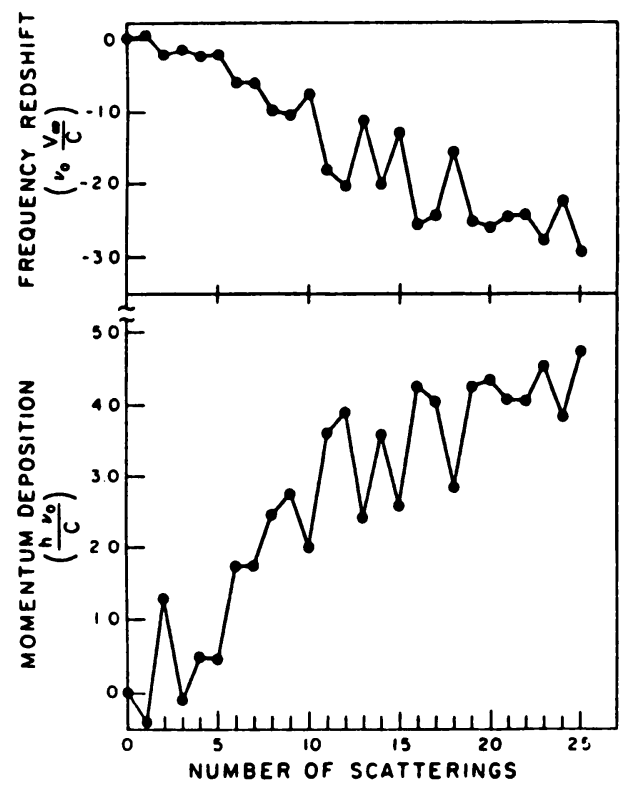

Figure 4. The cumulative frequency redshift (top panel) and momentum transfer (bottom panel), versus the number of the scatterings shown in Figure 3. (Abbott and Lucy 1985). 
winds continue to accelerate out to at least $4 \mathrm{R} *$. The evidence that there is acceleration out to $50 \mathrm{R} *$, found by Hillier (1987), now appears to be unnecessary or at least uncertain (Hillier 1990, these proceedings).

The effect of multi scattering is decreased if photons can escape between large $\Delta v$ gaps between thick lines. So, for the case of WR stars one wants to consider situations in which the line density vs. frequency is enhanced, as discussed in 2.4 .

\subsection{THE EFFECTS OF ANISOTROPIC DIFFUSE RADIATION FIELD}

In the usual Sobolev approach, e.g. as used in CAK, it is assumed that the continuum radiation field is anisotropic, i.e. coming from the direction of the star in a core-halo picture. It is this anisotropic field that gives rise to the outward force on the resonance shell. It is also assumed that because of multiple scattering within a single resonance shell that the diffuse line radiation is isotropic, and hence this radiation field provides no net outward force. However, for a thick Wolf-Rayet wind this latter assumption is a poor one, as has been shown by Puls and Hummer (1987). They considered the effects of a diffuse radiation field having an angular dependence $\propto \mu \mathrm{d} \mathrm{S} / \mathrm{dr}$, and find that this leads to an extra deposition of momentum close to the star. Figure 5 shows one of their results for the line acceleration. Because the deposition occurs deep in the wind, it gives rise to an increase in the wind mass loss rate, as we would expect from law I.

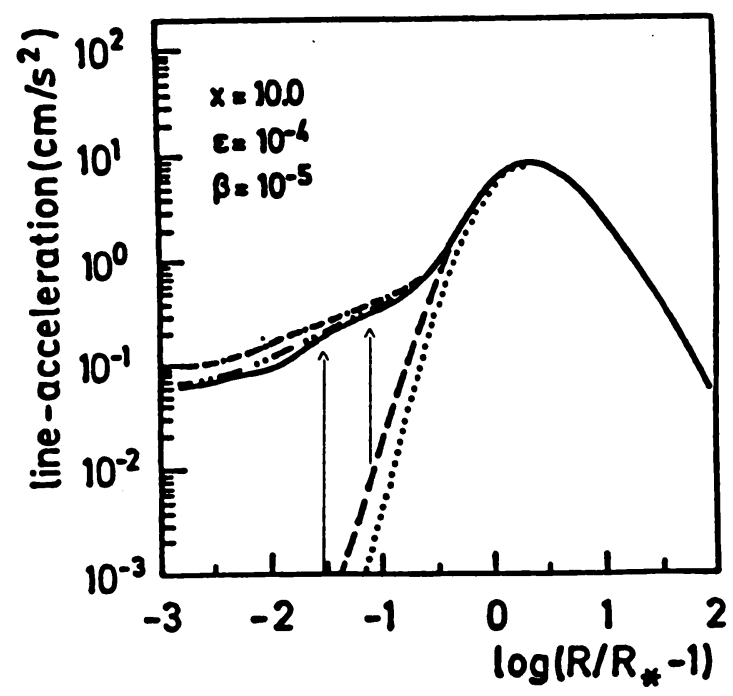

Figure 5. Shows the line acceleration versus logarithm of the height above the photosphere. The lower two curves show results of the Sobolev approximation, without accounting for the force associated with the diffuse radiation field in the line. The upper lines indicate the corrected acceleration, accounting for the diffuse radiation field. The arrows are drawn in to show the significant increase in acceleration that occurs at small heights. (Adapted from Puls and Hummer, 1988). 


\subsection{SMALL STAR EFFECTS}

There are more thick lines at higher wind densities, or equivalently for larger electron scattering Sobolev optical depths $=t=\sigma \rho \mathrm{v} /(\mathrm{dv} / \mathrm{dr})$. Recall that a basic feature of CAK theory is that the number of thick lines is proportional to a positive power of $t$. $(\mathrm{N}(\mathrm{t})=$ $\mathrm{k} \mathrm{t} \gamma$. Also, there are more strong lines in the EUV than in the near UV. Therefore, having Wolf-Rayet stars at a large $\mathrm{T}_{\text {eff }}(>50,000 \mathrm{~K})$ helps to explain the winds as radiatively driven. Abbott and Conti (1987) plot a boundary on the HR diagram to the left of which one can expect WR stars to be produced by multiple scattering effects. This line corresponds to an iso electron scattering line

$$
\begin{aligned}
\tau & =\text { Const }=\sigma_{\text {es }} \int \rho d r \\
& =\frac{.2 \dot{M}}{4 \pi v_{\infty}} \frac{1}{R} \ln \left(\frac{v_{\infty}}{v_{0}}\right)
\end{aligned}
$$

for which the Abbott and Lucy (1986) velocity law $(\beta=1)$ was used. Note that $\tau$ is increased as the stellar radius, $R *$, decreases. The line for constant $\tau$, is shown in Figure $6 \mathrm{a}$, and $6 \mathrm{~b}$ shows that for the WR evolutionary tracks of Maeder and Meynet WR stars do, in fact, enter the region where thick winds are expected.

In summary: we have discussed four radiative related effects that have come to light over the past decade. The bottom line is that it is only the multi-line effects that can really lead to an increase in the efficiency factor $\eta$ to values above unity. The "modified CAK" effects discussed in $\$ 4.1$ only redistribute the relative values of $\dot{M}$ and $v_{\infty}$, but do not change $\eta$ very much. We have seen that $\eta \gg 1$ can be derived numerically from the modified CAK model, but only by using unacceptable $\Gamma_{\text {es }}$ values, and because line overlap is not accounted for. The anisotropic diffuse field effects, discussed in § 4.3 correct for the core-halo approximation of CAK theories. Since the extra force is deep in the wind, the diffuse field forces tend to increase $\dot{M}$ according to law \# II. However, this does not really lend to an increase in the $\eta$ value because the flow must then be driven to high speeds, which cannot be done unless multi-line effects, or other forces, are accounted for. Thus we see that law \# II can be misleading. Let us consider other cases in which subsonic deposition occurs. The most important example is the radiative driving of the winds from Red Giants. Shocks propagating through the outer envelope "bloat" the atmosphere to the extent that the density is sufficiently high at the condensation radius that dust can form (Goldberg, 1987). At that point, radiative acceleration of grains drives the coupled dust/gas mixture out as a slow wind. Nonetheless, as we see in the paper by Jura (these proceedings) the $\eta$ values for these stars does not exceed unity. In regards to the Wolf-Rayet stars, it is suggested by interior theorists (Maeder, 1990; Langer 1990) that large mass loss rates are driven because the stars are near vibrational instability. The resultant disturbances would presumably affect the subsonic portion of the flow. However, a mechanism for driving the winds to high speed, such as the multi-line effects, would still be needed to explain values of $\eta$ greater then unity. We have seen in Owocki's paper (these proceedings) that if insufficient momentum is added to the supersonic part of the flow, the material will fall back onto the star.

It is important to note that the observational evidence for multi-line driving of winds with $\eta>1$ requires acceleration at large distances from the star. I hope that a major effort is placed on finding evidence for this acceleration in Wolf-Rayet stars.

Tremendous progress is being made by the Munich group in explaining the properties 

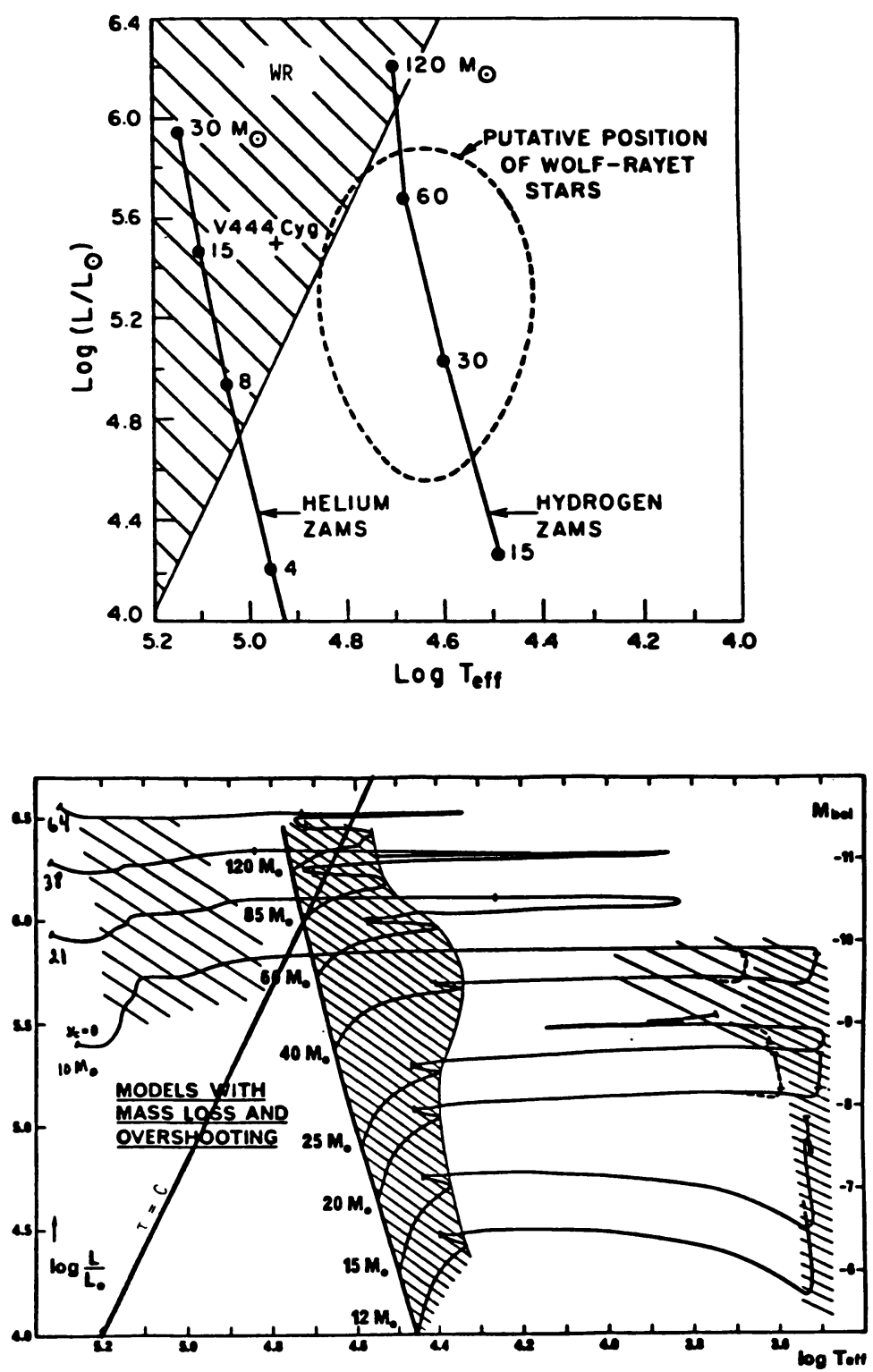

Figure 6. Hertzsprung-Russell diagrams showing properties of luminous stars.

(a) shows the hydrogen and helium zero-age main sequences (ZAMS). The shaded region shows where radiation driven winds are optically thick in the continuum. If W-R stars have the high temperatures and luminosities corresponding to the shaded region, the results of Abbott and Lucy (1985) indicate that their winds might be explained by radiation pressure alone, by way of multi-line effects. (From Abbott and Conti 1987). (b) shows the evolutionary tracks of Maeder and Meynet (1987). The dark line, (marked $\tau=C$ ) corresponds to the boundary shown in figure (6a). Thus we see that interior models indicate that stars should enter the region where multi-line effects are important. 
of radiation driven winds, and I refer you to the recent paper by Pauldrach et al. (1990).

As an introduction to the next section, it is important to realize the following: The multiple scattering should also be decreased if there is a non-spherical density distribution, such as an equatorially enhanced wind. This is because photons can more easily escape in the polar direction. Thus if observations show evidence for rotational distortion, not only is this evidence that another driving mechanism is operating, but the effect of radiative driving through multiple scattering is diminished.

\section{Luminous Magnetic Rotators}

As a second approach for explaining the Wolf-Rayet momentum problem, let us consider a non-spherical, rotating model. Wolf-Rayet stars are located in the HR diagram rather near to the hypergiant B[e] stars. Zickgraf et al. 1985 proposed that these stars have a two component structure: (1) a fast polar wind detected by the UV resonance lines of Si IV, C IV, N V, and which is presumably driven by the radiation field from the broad polar zone of the star and, (2) a slower, denser flow in the equatorial zone, where the state of ionization is lower and at some distance dust formation can occur. Poe, Friend, and Cassinelli (1989) developed a two component picture for Wolf-Rayet winds incorporating rotational and magnetic forces in the equatorial zone. Cassinelli (1990) has called this type of model a "Luminous Magnetic Rotator", because radiation forces play a major role in driving the flow. Such a picture for Wolf-Rayet stars has several advantages in regards to explaining some WR phenomena: (a) The model can explain the polarization that is seen in some WR stars (Schmidt 1988) and in B[e] stars (Zickgraf and Schulte-Ladbeck 1989). (b) The model provides an evolutionary link to the $\mathrm{B}[\mathrm{e}]$ and post-luminous blue variable stars. (c) It provides a structure conducive for the formation of dust grains that occurs in some B[e] and late WC stars. (d) The rotational model allows for an explanation of the Wolf-Rayet momentum problem, (e) and perhaps at a stellar "effective temperature" lower than that required for purely radiation driven winds. (f) A new "spin-down problem" is introduced, but this can be overcome with a properly chosen B field as shown by Poe et al. (1989).

Cassinelli, Schulte-Ladbeck, Poe and Abbott (1989) argued that the rapid rotator picture is at least plausible from an evolutionary point of view, and they showed results of a simple model to follow the evolutionary change in the ratio $\alpha=v_{\phi} / v_{\text {crit. }}$. The stars must be rotating with an equatorial velocity near the critical speed:

$$
v_{\text {crit }}=\sqrt{\frac{G M(1-\Gamma)}{R}}
$$

Near the Humphreys Davidson limit, stars lose about $40 \%$ of their mass before becoming sufficiently homogeneous to evolve to the left in the HR diagram. The models of Maeder and Meynet (1987) indicate that not only is the mass significantly smaller during the leftward evolution, but also the luminosity is increased. The change in $\mathrm{M}$ and $\mathrm{L}$ increases $\Gamma$ (equation 2). Hence $v_{\text {crit }}$ can be reduced by a factor of 2 relative to the rightward (pre LBV) phase of evolution. Since $v_{\phi}$ varies as (MR)-1, and $v_{\text {crit }}$ is smaller, $\alpha$ will be significantly closer to unity. In addition the paper by Linda Smith (these proceedings) shows that at least for some LBV's the mass loss appears to be bipolar. If the mass loss is in fact primarily from polar regions when stars are near the HD limit, the star can retain a larger angular momentum per unit mass, which makes the rapid rotator model more likely. The rapid rotator model is perhaps not needed for all Wolf-Rayet stars. 


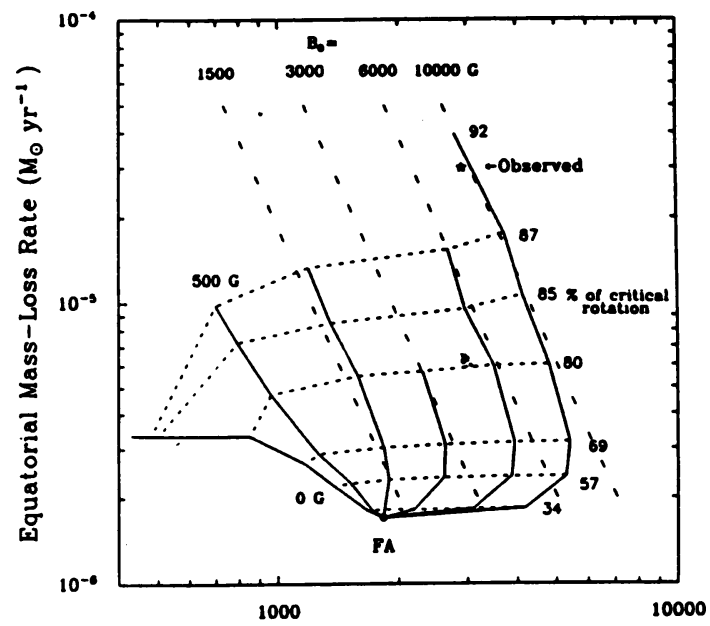

Figure 7. Shows the mass loss rates and terminal velocities that are expected for winds that are driven out of the equator by a combination of radiation pressure, magnetic, and rotational forces. (From Poe, Friend, and Cassinelli 1989). The equatorial mass loss shown is $(4 \pi) x$ the mass loss per unit solid angle in the equatorial region. The point FA indicates the $\mathbf{M}, v_{\infty}$ values derived in the modified CAK theory of Friend and Abbott (1986). The solid lines extending from the FA point are iso-magnetic field lines, ranging in values from 0 to 10,000 Gauss, as indicated. The short dashed, horizontal lines are iso-rotation rate (constant $\alpha$ ) lines. The point marked "observed" corresponds to the $M$, Vo determined for the WR star CV Ser from Radio and UV line profiles. The parameters for the models shown are: $M=13 M_{\Theta}, R_{\Theta}=8 R, \Gamma=0.36$.

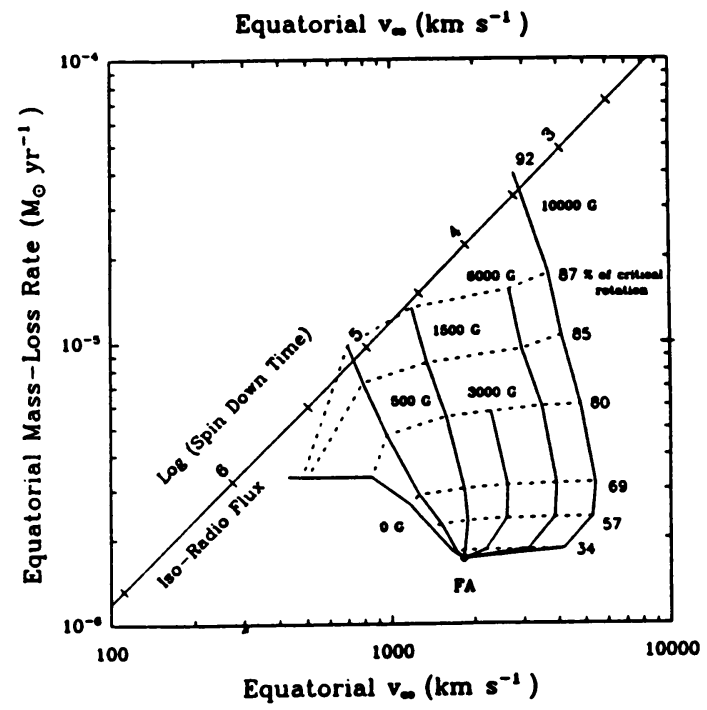

Figure 8: Same as Figure 7, but now contains a line of equal radio flux. This indicates that a wide range of models could potentially explain the observational estimates of $M$. Numbers along the iso-radio flux line are estimates of the logarithm of the spin down time of the star, which we see ranging from about $10^{3}$ yrs to over $10^{5}$ yrs. (From Poe et. al. 1989). 
Nonetheless, it is relevant for some stars and it is interesting to consider at least this one alternative to the purely radiation driven wind model.

Cassinelli (1990) points out that magnetic rotator wind models are always hybrid wind models, involving at least one additional driving mechanism, which is referred to as the primary mechanism. In the solar case the primary mechanism involves coronal forces, in the case of hot luminous stars it is the radiative driving. The primary mechanism sets the minimal mass loss rate for the star, and the magnetic rotator forces can change the speed of the wind and the mass loss rate. The basic theory for magnetic rotator winds was developed in a classic paper by Weber and Davis (1967). They showed that the wind momentum equation in the equatorial plane could be separated into purely radial and azmuthal $\left(v_{\phi}\right)$ equations, with associated wind energy and angular momentum constants.

As we have been discussing radiation driven winds in terms of momentum/energy deposition, it is useful here to discuss the magnetic rotator model in terms of the required deposition. The deposition, or transfer of energy from the magnetic field, occurs via the divergence of the Poynting vector, S, (Belcher and Mac Gregor, 1976) where

$$
\mathbf{S}=\frac{\mathbf{c}}{4 \pi}(\mathbf{E} \times \mathbf{B})
$$

where the electric field $\mathbf{E}$ is determined by the frozen-in magnetic field constraint. The total energy per gram, $\varepsilon$, is composed of a gas term plus the magnetic term,

$$
\varepsilon_{\text {mag }}=\frac{S_{r}}{\rho V_{r}}
$$

where $S_{r}$ is the radial component of the Poynting vector. Its value at infinity can be expressed in terms of a very convenient quantity, the Michel velocity, $\mathrm{V}_{\mathbf{M}}$;

$$
S_{r, \infty}=\rho V_{M}^{3}
$$

and $V_{M}$ is given in terms of the wind mass loss rate, the stellar rotation rate, stellar radius, and surface radial magnetic field as;

$$
\mathrm{V}_{\mathrm{M}}^{3}=\left(\frac{\Omega_{*}^{2} \mathrm{R}_{*}^{4} \mathrm{~B}_{\mathrm{r}, *}^{2}}{\dot{\mathrm{M}}}\right)
$$

This velocity plays a crucial role in magnetic rotator models. Let $V_{w}$ be the terminal wind speed associated with the primary wind forces. If $V_{M}>V_{W}$ there will be a significant transfer of energy from the field to the wind. This leads to a distinction between two of the three classes of magnetic rotator models. If $\mathrm{V}_{M}<\mathrm{V}_{\mathrm{w}}$, the star is a "Slow Magnetic Rotator" (SMR) (as is the current sun). If $V_{M}>V_{w}$, the winds radial velocity is affected by the field, and the star is a "Fast Magnetic Rotator" (FMR). The separation occurs at

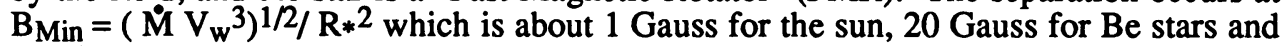
300 for WR stars. For sufficiently rapid rotation, a third magnetic rotator domain exists, 
the "Centrifugal Magnetic Rotator" (CMR), a name used by Cassinelli (1990), but discussed as an "extreme FMR" by Hartmann and Mac Gregor (1980). In a CMR, (a) the terminal speed is $\mathrm{V}_{\infty} \approx \mathrm{V}_{\mathbf{M}}$, (b) the equatorial subsonic region is in solid body co-rotation with the star, and (c) the sonic point occurs where $V_{\phi}$ (solid body) $=V$ (circular) or

$$
r_{s} \Omega_{*}=\sqrt{\frac{G M}{r_{s}}}
$$

Thus, we have one of the simplest results for a critical point in all of wind theory (!)

$$
\left(r_{S} / R *\right)=1 / \alpha^{2 / 3}
$$

In this CMR case, $\rho(r)$ in the subsonic region is determined by gravity and centrifugal forces and is independent of the field. The CMR winds thus provide for a nice clean separation between $\Omega$ effects and B field effects. The mass loss rate depends only on $\alpha=$ $\Omega / \Omega_{\text {crit, }}$, and given this $\dot{M}$ the terminal velocity $\left(=V_{m}\right)$,is determined by $B_{r}, *$. Figure 7 shows a result of Poe et al. for the Wolf-Rayet star CV Ser. Various iso-V $\mathbf{M}_{M}$ lines are labeled with the surface field. The point marked FA corresponds to the Friend Abbott (Modified CAK) result for this star. This provides a minimal mass loss rate that is far below the radio estimate labeled "observed". Shown by what I call a flower diagram are the results of luminous magnetic rotator models for various assumed base fields $B_{r}{ }^{*}$ and rotation rate $\alpha$ ratios. Note that the centrifugal domain, in which the mass loss rate increases, begins for $\alpha$ above about 34\% maximum. To achieve the "observed" point would require a field of $10^{4}$ Gauss and $\alpha=0.90$, as was derived by Hartmann and Cassinelli (1981) [discussed by Cassinelli 1982]. Their solution has several problems. The magnetic field is too large, i.e. would drastically modify the hydrostatic structure of the atmosphere. Secondly, the model suffers from a "spin-down problem", with a rotation e-folding time far below the $\approx 10^{5}$ year lifetime of Wolf-Rayet stars. Figure 8 from Poe et al. illustrates some of the problems and possible solutions. The diagonal line corresponds to equatorial models that provide the observed radio flux, from which the mass loss rates are derived. This shows that one can explain the radio flux with a range of models, such as a high speed wind with $B=10^{4}$ Gauss and $\dot{M}=3 \times 10^{-5} \mathrm{M} / \mathrm{yr}$ or with a slower equatorial wind with $B \approx 1000$ Gauss with $\stackrel{M}{M}=10^{-5} \mathrm{M} / \mathrm{yr}$. The numbers on the iso-radio flux line give the spin down times. Thus note that the $10^{4}$ gauss model corresponds to a spin down time of only a few thousand years and therefore is unacceptable. Figure 8 also indicates that a wide range of $B, \hat{M}, v_{\infty}$ are possible. It would be useful to narrow the range of say the B field by requiring that the field be consistent with stellar interior constraints. This requirement is analogous to our new rule for the purely radiative driven models, that $\Gamma$ be consistent with interior values.

Maheswaran and Cassinelli (1988) recently derived constraints on the surface fields in rapidly rotating stars. These are shown in Figure 9, in which is plotted B versus the rotation ratio $\alpha$. Rapid rotation leads to circulation currents in the interior. If the current speed is larger than the Alfvén speed, the surface magnetic field will be effectively submerged. Hence, the surface field is either approximately zero or it is sufficient to overcome the submersion effect and is above $B_{\min }$, that is shown on the Figure. If the field is very large it dominates the hydrostatic equilibrium equation, and by analogy with radiation driven theory we can say the field can exceed a Magnetic Eddington Limit, also shown on the Figure as $B_{\max }$. These limits, either zero or within the triangular allowed zone, are now plotted on the $M$ versus $v_{\infty}$ plane, and form what I call a "cat" diagram in Figure 10. 


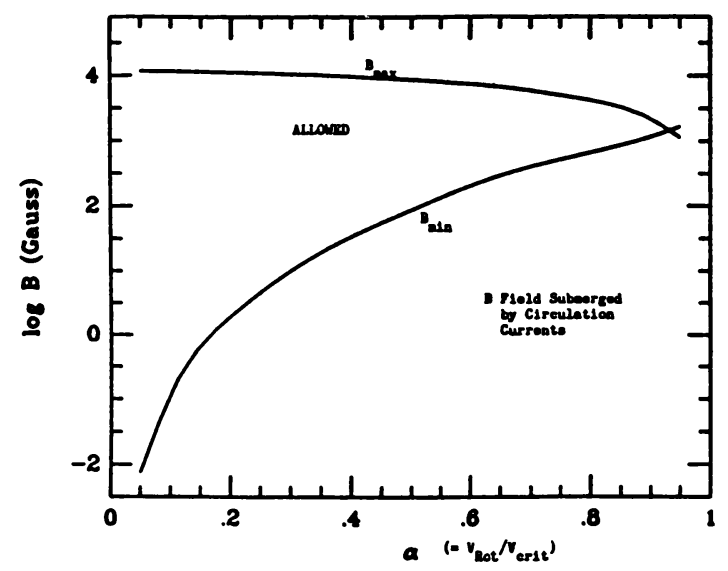

Figure 9: Shows surface magnetic fields versus rotation rate parameter $\alpha\left(=V_{\phi} / V_{\text {crit }}\right)$. The roughly triangular zone corresponds to the allowed values of the magnetic field for the star discussed in Figures 7 and 8 . If the field is above $B_{\max }$, the hydrostatic structure is dominated by the magnetic field/rotation forces. If the field is below $B_{\min }$, the circulation currents have a velocity greater than the Alfven speed in the outer stellar envelope, and the field will be submerged. The surface field must either be approximately zero, or be in the "allowed region". (From Maheswaran and Cassinelli, 1988).

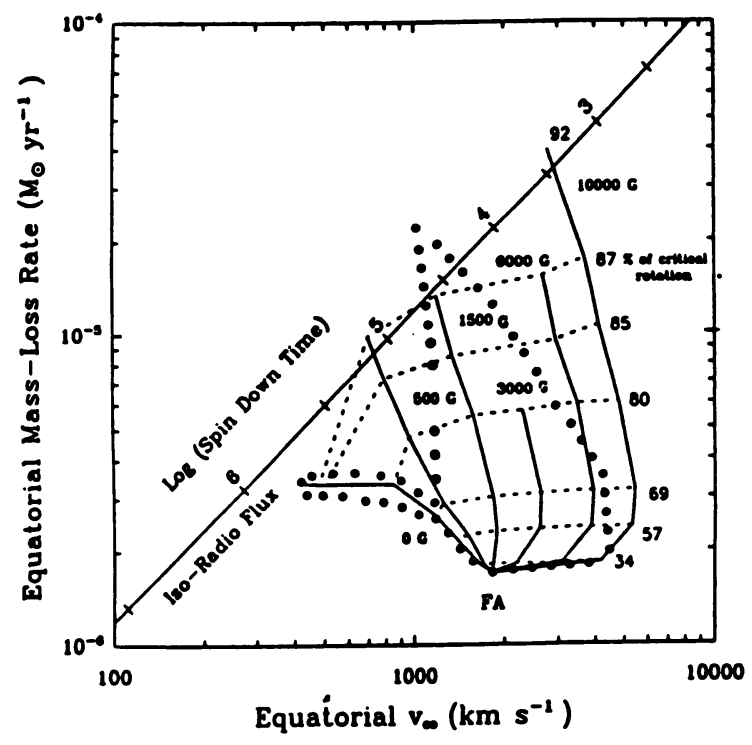

Figure 10. Same as figure 8, but here the dark dots indicated the "Allowed" range of magnetic fields as derived from Figure 9. Note that the Allowed region includes a portion of the iso-radio flux line, with a not-implausible spin down rate. 
Thus we have narrowed the allowable range of stellar parameters and it includes a portion of the iso-radio flux line, with a not implausible spin down time. Various parameters associated with the FA point can be adjusted so that the actual length along the iso-radio line could be somewhat larger.

In summary; the rotational model provides an explanation of the momentum problem as follows: the product of $\dot{M} v_{\infty}$ is made up of two parts which are derived from two quite different parts of the stellar wind. The Wolf-Rayet mass loss rates are derived from radio fluxes and this radiation arises primarily from the denser, slower equatorial regions of a Luminous Magnetic Rotator. The terminal velocity, $v_{\infty}$, is derived observationally from $P$ Cygni line widths, which are determined by the highest speed along the line of sight in front of the star. The wind outside the narrow equatorial zone is radiatively driven to high speeds, so it is this "polar flow" that determines $v_{\infty}$. Neither the polar nor equatorial flow violate the momentum maximum $\eta \approx 1$. The momentum problem is thus solved in a piece-wise fashion. This general idea that the high radio flux does not necessarily mean a high mass loss rate should be applicable to other non-spherical pictures such as the cloud model discussed by Cherapascheck and Nugis elsewhere in these proceedings.

\section{Summary and Conclusions}

In this paper we have discussed two approaches for finding a solution to the Wolf-Rayet momentum problem; purely radiative winds, and luminous magnetic rotators. In regards to the purely radiative wind research, we have discussed four line driven wind effects. The solution of the momentum problem requires the multi-line effects that have been analyzed most thoroughly by Puls (1987). The observational signature of the multi-line radiative driving is the continued acceleration of the flow at large radial distances. Hillier (1987) has described some observational evidence for such acceleration. Also, the observed saturated UV P Cygni lines of Wolf-Rayet stars do not have a sharp violet edge, but rather a gradual rise from zero intensity up to the continuum level (Prinja et al. 1990). Currently this is explained as being caused by absorption in shocks with speeds as high as $1000 \mathrm{~km} / \mathrm{sec}$. Perhaps part or most of that high velocity absorption could be caused in the distant acceleration from multi-line effects. It is very important that evidence for the distant acceleration be searched for.

If the multi-line mechanism cannot explain the momentum problem, then clearly we need to look at the alternative models. The best evidence for rotational distortion is the presence of intrinsic polarization and characteristic changes in polarization across strong emission lines (Schmidt, 1988; Schulte-Ladbeck, 1990). Here we have presented some arguments indicating that rapid rotation of Wolf-Rayet stars is a plausible outcome of their evolution. The luminous magnetic rotator model, explains the momentum problem by having the high density and high velocity portions of the wind occuring in different places. Stellar interior theorists favor the vibrational instability mechanisms for driving mass loss. However, from the point of view of stellar wind theory, it is not at all clear how this can lead to the high speed outflows without also utilizing the multi-line acceleration mechanism.

The main conclusions that I would like to stress are that: a) there are at least two ways to explain the Wolf-Rayet momentum problem. Neither theory is, as yet, fully worked out, b) the models have observational consequences that can and should be searched for. 


\section{References}

Abbott, D.C. (1982), Ap.J., 263, 723.

Abbott, D.C. (1988), in proceedings of the 6th Solar Wind Conference NCAR TN 306, eds. V.C. Pizzo, T.E. Holzer, D.G. Sime (NCAR), p. 149.

Abbott, D.C. and Conti, P.S. (1987), Ann. Rev. Ast. Astrophys., 25, 113.

Abbott, D.C. and Lucy, L.B. (1985), Ap. J., 288, 679.

Barlow, M.J., Smith, L.J. and Willis, A.J. (1981), M.N.R.A.S., 196, 101.

Belcher, J.W. and MacGregor, K.B. (1976), Ap.J., 268, 498.

Cassinelli, J.P. (1982), in IAU Symp. 99. Wolf-Rayet Stars: Observations. Physics and

Evolution, eds. C.W.H. de Loore and A.J. Willis, (Dordrecht: Reidel), p. 173.

Cassinelli, J.P. (1990), to appear in Angular Momentum and Mass Loss from Hot Stars, eds. L.A. Willson and R. Stalio, (Kluwer: Dordrecht).

Cassinelli, J.P. and Castor, J.I. (1973), Ap.J., 179, 189.

Cassinelli, J.P., Schulte-Ladbeck, R.E., Poe, C.H., and Abbott , M. (1989), in IAU Colloq. 113. Physics of Luminous Blue Variables, eds. K. Davidson, H.J.G.L.M.

Lamers, and A.F.J. Moffatt, (Kluwer: Dordrecht), p. 121.

Castor, J.I., Abbott, D.C., and Klein, R.I. (1975), Ap.J., 195, 157.

Friend, D.B. and Abbott, D.C. (1986), Ap.J., 311, 701.

Friend, D.B. and Castor, J.I. (1983), Ap.J., 272, 259.

Friend, D.B., Poe, C.H., and Cassinelli, J.P. (1987), B. A. A. S., 20, 1012.

Goldberg, L. (1987), in The M Type Stars, NASA SP-492, eds. H.R. Johnson and F.R. Querci, p. 245.

Hartmann, L. and Cassinelli, J.P. (1981), B. A. A. S., 13, 795.

Hartmann, L. and MacGregor, K.B. (1982), Ap. J., 259, 180.

Hillier, D.J. (1987), Ap.J. (Suppl.), 63, 965.

Koenigsberger, G. (1990), these proceedings.

Langer, N. (1990), these proceedings.

Leer, E. and Holzer, T.E. (1980), J.Geophys. Res., 85, 4681.

Lucy, L.B. and Soloman, P.M. (1970), Ap.J., 159, 879.

Maeder, A. and Meynet, G. (1987), Astron. Astrophys., 182, 243.

Maeder, A. (1990), these proceedings.

Maheswaran, M. and Cassinelli, J.P. (1988), Ap.J., 335, 931.

Panagia, N. and Macchetto, F. (1981), in IAU Colloq. 59. Effects of Mass Loss in Stellar Evolution, eds. C. Chiosi and R. Stalio (Dordrecht: Reidel), p. 173.

Pauldrach, A., Puls, J., Hummer, D.G., and Kudritzki, R.P. (1985), Astron. Astrophys., 148, L1.

Pauldrach, A., Puls, J., Kudritzki, R.P. (1986), Astron. Astrophys., 164, 86.

Pauldrach, A., Kudritzki, R.P. Puls, J., and Butler, K. (1990), Astron. Astrophys., 228, 125.

Poe, C.H., Friend, D.B., and Cassinelli, J.P. (1989), Ap.J., 337, 888.

Prinja, R.K., Barlow, M.J., and Howarth, I.D. (1990), Ap. J.,

Puls, J. and Hummer, D.G. (1988), Astron. Astrophys., 191, 87.

Puls, J. (1987), Astron. Astrophys., 184, 227.

Schmidt, G.D. (1988), in Polarized Radiation of Circumstellar Origin, eds. G.V. Coyne et al., (Vatican Observatory: Vatican), p. 641

Schmutz, W., Harmann, W.-R., Wessolowski, U. (1989), Astron. Astrophys., 210, 236.

Schulte-Ladbeck, R.E. (1990), these proceedings.

Weber, E.J., and Davis, L. Jr. (1967), Ap.J., 268, 228.

Zickgraf, F.J. and Schulte-Ladbeck, R.E. (1989), Astron. Astrophys., 214, 274. 


\section{DISCUSSION}

Conti: I will be ready to agree that rotation may play some role in WR stars. But how important is it physically? One star we know the rotation of is HD 50896 (from the periodic polarization changes). Its rotation period is 3.76 days, implying a very slow equatorial velocity. You might see what effect such an equatorial velocity actually does to HD 50896 . I would think it small. It cannot by itself create the non-spherical shape of HD 50896.

Cassinelli: It appears from some of the poster papers that we really do not understand the nature of the 3.76 day resonance seen in HD 50896. In fact there is some evidence for a period of $1 / 3$ of that. So I think the possibility of rapid rotation is still open. Secondly, the polarization QU plot of the HeII 4686 line in HD 50896 shows a loop if you go from one side of the line to another. This is good evidence for a disk - i.e. material that exists all around the star and not just on one side towards a possible companion.

Sreenivasan: These massive stars have strong winds which spin down the surface layers rapidly to zero well before hydrogen is exhausted in the core. Angular momentum transferred from the core to the surface is also carried away efficiently. Magnetic fields, if present, will also very effectively slow down the star (e.g. the Sun). So the Michel velocity would, I am afraid, not help very much. (It does in the case of pulsars). Have you actually checked that this is not a problem? The co-rotation radius (where field lines rotate at the speed) is quite far away and would not be below the sonic point!

Cassinelli: Yes, we have done some modelling of the rotation rate vs. time, Cassinelli et al. (1989). The B[e] stars could only be explained as occurring after the mass loss at the Humphreys-Davidson limit, at which the stars eject $\sim 40 \%$ of their mass and $\Gamma$, allowing the post HD evolution to occur with a smaller maximal rotation speed $\sqrt{G M(1-\Gamma)} / R$. It of course helps tremendously if the mass loss at the HD limit occurs primarily at the poles. Now in regards to your second comment, recall that the Maheswaran limits say that the field is either zero or above a certain limit. So one can have a post-main-sequence/preHumphreys-Davidson limit occur with zero field, so as to minimize the angular momentum loss. The mass ejection at the HD limit would of course expose the deeper layers of the star and magnetic effects might become important only after this phase. The fact that $B[e]$ stars occur in the pre-WR location in the HR diagram makes this picture plausible. The picture is very complicated both from the point of view of mass loss evolution and surface magnetic field evolution.

Koenigsberger: (1) I have a poster outside on HD 193077, where I claim to see the gravity darkening effect pretty good where you have a rapidly rotating star. Regardless of whether we believe my photospheric absorptions or not, Massey (1980) pretty much concluded that the $\mathrm{O}$ star spectrum in the optical region is definitely rotationally broadened to $550 \mathrm{~km} \cdot \mathrm{s}^{-1}$. This is much larger than any $\mathrm{O}$ star measured by Conti and Ebbets. So, definitely there is evidence for rapid rotation in HD 193077. (2) (question to De Greve) When you have close binary systems we assume that they are co-rotating. So, if you start out by two $O$ stars which are co-rotating, and one of them goes to a WR star, we are talking about rotational velocity periods of about three days, if this co-rotation is maintained into the WR phase. This is something no evolutionary model has taken into account.

Cassinelli: (1) It is good to know that there is evidence for rapid rotation of WR stars. It is often thought that WR stars should be slow rotators. (2) The thing about these very luminous stars is that they do not live very long, so a lot of these co-rotation effects might not be important. The other thing is, it happens when a star is undergoing very rapid phases in evolution like shrinking when it is going to the left into the HR diagram, it is collapsing, so it is going to spin up. So, even if it had been locked at some point earlier, it is going to get delocked and spin up. 
Montmerle: If the magnetic rotator model works for WR stars, it should also play a role in the $\mathrm{O}$ star progenitor, i.e., over the preceding few $10^{6}$ years. Then one can expect a very efficient spin down (note: magnetic fields must be present in $\mathrm{O}$ stars, since many of them are non-thermal radio emitters).

Cassinelli: We would have to argue, from the Maheswaran results, that the surface field is small or zero during the $\mathbf{O}$ star phase of evolution, i.e. the circulation currents submerge the surface field, while the stars are in the pre-HD phase. I will admit that there must be some field in the winds of some $\mathbf{O}$ stars. We should consider how that occurs.

Walborn: One property of $\mathrm{B}[\mathrm{e}]$ stars is that, unlike OB supergiants and LBVs, they show no evidence of CNO anomalies, so I seriously doubt that they are very highly evolved objects or are related to WR stars.

Cassinelli: That is an interesting point. It is the location of the B[e] stars in the HR diagram that we have tried to explain. The stars are very luminous and well separated from the main sequence, and show evidence for a two-component structure, fast polar flow and slower, denser equatorial flow (the Zickgraf model). It is difficult to explain this except as an phenomenon that occurs as post-HD limit stars.

Hamann: If WR winds would be less dense, but faster at the poles, compared to equatorial regions, this would severely affect the formation of the spectral lines. However, mass loss rates we derive from detailed fitting of line profiles by means of spherically symmetric models are in excellent agreement (within $\pm 25 \%$ ) with the rates derived from the radio emission. In my opinion, this rules out any drastical deviations from spherical symmetry. Cassinelli: I think that one could also fit line profiles and radio fluxes with a non spherical picture. For example, Rumpl (1980) was able to get excellent fits to the UV line profiles of HD 50896 using a model in which the density in the equatorial zone greatly enhanced. Another thing I would like to stress is that there should be a significant flow out the polar regions, i.e. the winds would be as strong as, say, Of stars, out the polar zone. So you should not picture these stars as having mass outflow primarily in the equatorial zone, as is commonly envisioned for the Be stars. Finally, I think that there are enough adjustable parameters that one could fit both the UV line profiles and radio fluxes as well as does your spherical models. But of course there is a need to really do the calculations to verify this statement.

Barlow: If the mass loss rates of most WR stars are strongly influenced by rotation, why is HD 50896 the only "single" WR star which shows a well-defined period plus the polarization effects indicative of an asymmetric mass distribution?

Cassinelli: There are other stars that show intrinsic polarization effects. However, perhaps one reason why more WR stars do not show larger polarization is because the polar flow is not insignificant. The stars are not like $\mathrm{Be}$ stars in which there is negligible mass loss in polar regions. These stars have strong polar winds. We assume that the polar zone winds satisfy the radiation momentum limit, but as we know from the OB stars this corresponds to a significant wind. The point is that the polarization can be reduced because of cancellation that occurs because of scattering from electrons in the polar zone. In $\mathrm{Be}$ stars that cancellation effect does not occur.

Lozinskaya: I would like to remind you that the X-ray image of the WR star in NGC 6888 looks like this: we see two X-rays spokes inside the bubble, which may be considered as an indication of bi-polar wind. 


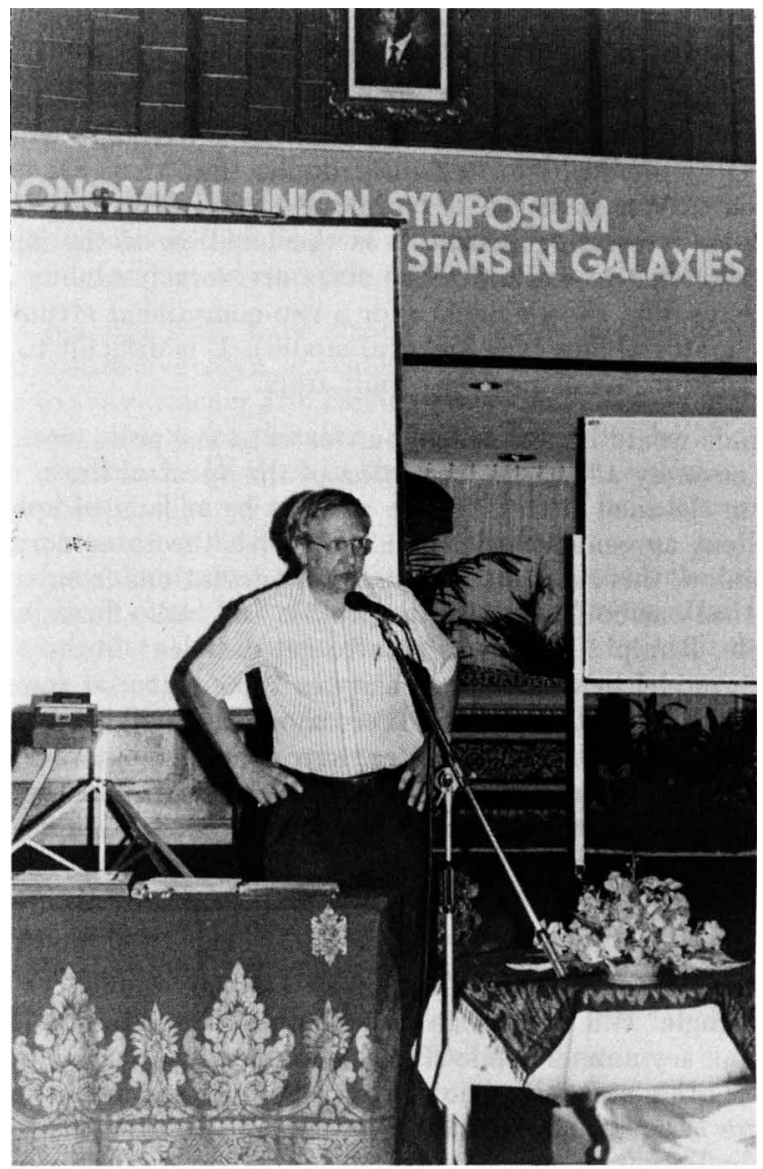

Joe Cassinelli 